\title{
Lithocarpus polystachyus Rehd. leaves aqueous extract protects against hydrogen peroxide-induced SH-SY5Y cells injury through activation of Sirt3 signaling pathway
}

\author{
JIANMEI GAO ${ }^{1}$, YINGSHU XU ${ }^{1}$, JIANYONG ZHANG ${ }^{1}$, JINGSHAN SHI $^{2,3}$ and QIHAI GONG ${ }^{2,3}$ \\ ${ }^{1}$ Department of Clinical Pharmacotherapeutics, School of Pharmacy, Zunyi Medical University; \\ ${ }^{2}$ Department of Pharmacology, Key Laboratory of Basic Pharmacology of Ministry of Education; \\ ${ }^{3}$ Joint International Research Laboratory of Ethnomedicine of Ministry of Education, \\ Zunyi Medical University, Zunyi, Guizhou 563000, P.R. China
}

Received June 30, 2017; Accepted September 17, 2018

DOI: $10.3892 /$ ijmm.2018.3916

\begin{abstract}
Lithocarpus polystachyus Rehd. (sweet tea; ST) leaves is a type of Chinese folkloric medicine from southern China. The purpose of the present study was to explore the neuroprotective effect of ST, and to explore its underlying mechanisms in hydrogen peroxide $\left(\mathrm{H}_{2} \mathrm{O}_{2}\right)$-induced neuronal cell injury in cultured human neuroblastoma. $\mathrm{H}_{2} \mathrm{O}_{2}$ was used as oxidant inducer and human SH-SY5Y neuroblastoma cells were treated with various concentrations of ST. Cell viability and cell death were detected using MTT and LDH assays, respectively. Additionally, the production of intracellular and mitochondrial reactive oxygen species (ROS) were determined by 2',7'-dichlorodihydrofluorescein diacetate (DCFH-DA) and MitoSOX Red, respectively. The production of malondialdehyde (MDA), reduced glutathione (GSH) level, glutathione peroxidase (GSH-Px), superoxide dismutase (SOD) activities, and $\mathrm{NAD}^{+} / \mathrm{NADH}$ ratio were confirmed using relevant kits. The expression of adenosine monophosphate-activated protein kinase (AMPK), peroxisome proliferator-activated receptor coactivator (PGC)-1 $\alpha$, Sirt3, isocitrate dehydrogenase (IDH)2, forkhead boxO3a (Foxo3a), and SOD2 were analyzed by western blot analysis. It was demonstrated that
\end{abstract}

Correspondence to: Professor Qihai Gong, Department of Pharmacology, Key Laboratory of Basic Pharmacology of Ministry of Education, Zunyi Medical University, 6 Xuefu West Road, Zunyi, Guizhou 563000, P.R. China

E-mail: gqh@zmc.edu.cn

Abbreviations: ROS, reactive oxygen species; DCFH-DA, 2',7'-dichlorofluorescin diacetate; MDA, malondialdehyde; SOD, superoxide dismutase; GSH, reduced glutathione; GSH-Px, glutathione peroxidase; AMPK, adenosine monophosphate-activated protein kinase; PGC-1 $\alpha$, peroxisome proliferator-activated receptor coactivator-1 $\alpha$; Foxo3a, forkhead box O3a

Key words: SH-SY5Y cell, hydrogen peroxide, reactive oxygen species, neurodegenerative disease pre-treatment with ST enhanced cell viability and repressed cell death, and it also reduced intracellular and mitochondrial ROS accumulation. Additionally, ST attenuated MDA production and enhanced GSH level, GSH-Px and SOD activities. Furthermore, ST not only increased $\mathrm{NAD}^{+} / \mathrm{NADH}$ ratio, but also inhibited the decrease of AMPK, PGC-1 $\alpha$, Sirt3, IDH2, Foxo3a, and SOD2. The present study revealed that ST exerts protective effects against oxidative stress-induced SH-SY5Y cells injury, and the underlying mechanisms are, at least partly, associated with its antioxidant capacity and function through mitochondrial Sirt3 signaling pathway.

\section{Introduction}

Oxidative stress is one of the main causes of neuronal injury in neurodegenerative disorders, involving different signaling pathways (1). Evidence declares that reactive oxygen species (ROS) or hydrogen peroxide $\left(\mathrm{H}_{2} \mathrm{O}_{2}\right)$, a main precursor of $\mathrm{ROS}$, induces neuronal cell death contributing to the pathogenesis in neurodegenerative disorders such as Alzheimer's and Parkinson's diseases and stroke (2). Neuronal cells respond to $\mathrm{H}_{2} \mathrm{O}_{2}$ with cytotoxicity and neurodegeneration, whereas antioxidants may abolish these effects (3). Notably, mitochondria is the major source of ROS and mitochondrial dysfunction has been recognized as a critical contributor to the neurodegenerative disorders (4). Furthermore, the antioxidant defense system serves vital role in regulating oxidative stress, and Chinese medicinal herbs provide rich resources for developing natural antioxidants (5). Therefore, it is important to elucidate antioxidants from natural products and herbal medicine for treating neurodegenerative disorders.

Lithocarpus polystachyus Rehd. is distributed as a wild plant in the mountain area in Southern China, especially in Shiqian and Fenggang cities of the Guizhou Province with plentiful natural resources (6). It is also called 'Sweet Tea' (ST) and its leaves are used as a medicinal tea and a folk herbal medicine to prevent or cure multiple diseases for many years and exhibited no adverse drug reactions or toxic effects (7). Accumulating evidence demonstrates that ST has extensive pharmacological activities, including anti-hypertensive, 
anti-hypoglycemic, anti-diabetic and anti-obesity pharmacological effects (7-9). However, little is known about whether ST possesses protective effects on the oxidative stress-induced neuronal cell injury.

AMP-activated protein kinase (AMPK) is known as a vital intracellular serine/threonine protein kinase that regulates celluar energy metabolism and responses to metabolic stress (10). Additionally, AMPK-activated peroxisome proliferator-activated receptor coactivator-1 $\alpha$ (PGC-1 $\alpha$ ) exerts crucial effect in the regulation of mitochondrial biogenesis and oxidative stress (11). Sirtuins belong to class III histone deacetylases family and their enzymatic activities are dependent on nicotinamide adenine dinucleotide, and seven mammalian homologues of sirtuins (Sirt1-7) have been identified (12). Among those sirtuins, Sirt3 has been demonstrated to control mitochondrial oxidative signaling, and serves as the downstream target of AMPK-PGC-1 $\alpha$ signaling pathway $(13,14)$. Additionally, a previous study reported that Sirt3 activation prevents neuronal mitochondrial oxidative stress and damage in neurodegenerative disorders (15). However, whether ST regulates neuronal Sirt3 and its corresponding upstream and downstream regulatory signaling pathways remain unclear.

Therefore, the present study was designed to determine the protective effect of ST on SH-SY5Y cell injury induced by $\mathrm{H}_{2} \mathrm{O}_{2}$, and to further elucidate its underlying mechanisms.

\section{Materials and methods}

Materials. Dulbecco's modified Eagle's medium: Nutrient Mixture F-12 (DMEM/F-12) (cat. no. 11320082), fetal bovine serum (FBS; cat. no. 1618862) and a penicillin/streptomycin mixture (cat. no. 15070-063) were obtained from Gibco; Thermo Fisher Scientific, Inc. (Waltham, MA, USA). 3-(4,5-dimethyl thiazol-2-yl)-2,5-diphenyl tetrazolium bromide (MTT; cat. no. M2128), 2',7'-dichlorofluorescin diacetate (DCFH-DA; cat. no. D6883), and rhodamine 123 (R8004) were obtained from Sigma-Aldrich; Merck KGaA (Darmstadt, Germany). MitoSOX Red Mitochondrial Superoxide Indicator (M36008) and Mito Tracker green probe (M7514) were obtained from Invitrogen; Thermo Fisher Scientific, Inc. The lactate dehydrogenase (LDH; cat. no. A020-2), malondialdehyde (MDA; cat. no. A003-1), superoxide dismutase (SOD; cat. no. A001-3), reduced glutathione (GSH; cat. no. A006-2) and glutathione peroxidase (GSH-Px; cat. no. A005) assay kits were purchased by Nanjing Jiancheng Bioengineering Institute (Nanjing, China). Anti-adenosine monophosphate-activated protein kinase (AMP; cat. no. ab131512), anti-p-AMPK (cat. no. ab23875), anti-peroxisome proliferator-activated receptor coactivator-1 $\alpha$ (PGC-1 $\alpha$; cat. no. ab54481), anti-Sirt3 (cat. no. ab217319), anti-isocitrate dehydrogenase (IDH2; cat. no. ab84726), anti-forkhead boxO3a (Foxo3a; cat. no. ab12162) and anti-SOD2 (cat. no. ab13533) antibodies were obtained from purchased from Abcam (Cambridge, UK). All other chemicals were of analytical grade.

Preparation of ST aqueous extracts. Fresh leaves of ST were obtained from Fenggang City in Guizhou Province in summer and identified by Professor Jianwen Yang (School of Pharmacy, Zunyi Medical University, Zunyi, China). A voucher specimen was deposited at the herbarium of School of Pharmacy, Zunyi
Medical University (voucher number, 20151016ST). From $1 \mathrm{~kg}$ dried ST, $100 \mathrm{~g}$ of dried aqueous extract was obtained (yield 10\%) and filtered. Subsequently, the filter liquor were condensed by rotary vacuum evaporation and lyophilized. The final powder was dissolved in double-distilled water, sterilized by passing through $0.22 \mu \mathrm{m}$ filters, and attenuated in DMEM/F-12 culture solution prior to employ at a final concentration of 25,50 or $75 \mu \mathrm{g}$ raw herb/ml. ST was standardized by the content of phlorizin and trilobatin, which are the active components of ST (16). Phlorizin and trilobatin (Chengdu Push Bio-Technology Co., Ltd., Chengdu, China) were determined by reverse-phase high-performance liquid chromatography (HPLC; Agilent 1200 series; Agilent Technologies, Inc., Santa Clara, CA, USA) with a UV detector, auto-sampler, column oven, and multi-channel pump. Subsequently, the separation was executed by a Phenomenex Luna end-capped C18 column $\left(5 \mathrm{~mm}, 4.6 \times 250 \mathrm{~mm}\right.$; Phenomenex, CA, USA) at $25^{\circ} \mathrm{C}$. The detector wavelength was set at $285 \mathrm{~nm}$. The separated conditions of phlorizin were as follows: Acetonitrile: Water (30:70, $\mathrm{v} / \mathrm{v})$ as mobile phase using a linear gradient at a flow rate of $1.0 \mathrm{ml} / \mathrm{min}$. The ST contained $1.19 \%$ phlorizin (Fig. 1A and $\mathrm{B}$ ). The separated conditions of trilobatin were as follows: Acetic acid water: Methanol (40:60, v/v) as mobile phase using a linear gradient at a flow rate of $1.0 \mathrm{ml} / \mathrm{min}$. The ST contained $18.54 \%$ trilobatin (Fig. 1C and D).

Cell culture. The human neuroblastoma SH-SY5Y cells were purchased from American Type Culture Collection (Manassas, VA, USA) and maintained in DMEM/F-12 supplemented with $10 \% \mathrm{FBS}$ and $0.1 \%$ penicillin/streptomycin at $37^{\circ} \mathrm{C}$ in a humidified incubator containing $5 \% \mathrm{CO}_{2}$ and $95 \%$ air.

MTT assay. Effect of ST on SH-SY5Y cell viability were determined using an MTT assay. In brief, SH-SY5Y cells were cultured in 96-well plates, at a density of $2 \times 10^{4}$ cells per well for $24 \mathrm{~h}$ at $37^{\circ} \mathrm{C}$. Subsequently, the cells were pre-treated with various concentrations $(25,50$ and $75 \mu \mathrm{g} / \mathrm{ml})$ of ST aqueous extracts or NAC for $1 \mathrm{~h}$ and co-treatment with $200 \mu \mathrm{M} \mathrm{H}_{2} \mathrm{O}_{2}$ for the further $24 \mathrm{~h}$ at $37^{\circ} \mathrm{C}$. Following the treatment, MTT solution was added to each well and the cells were cultured for $4 \mathrm{~h}$ at $37^{\circ} \mathrm{C}$. The dark-blue formazan crystals were dissolved in DMSO, and the absorbance values were detected at $490 \mathrm{~nm}$ wavelength by microplate reader. In addition, according to our primary study, $20 \mu \mathrm{M}$ NAC was chosen to be a positive control for antioxidant capacity that suppresses cell death for comparing with the possible protective effect of ST (17).

LDH leakage assay. The SH-SY5Y cells $\left(2 \times 10^{4}\right.$ cells per well) were pre-treated with or without ST as described above and the supernatant was analyzed by a LDH detection kit, according to the manufacturer's protocol. The leakage of LDH was determined at $490 \mathrm{~nm}$ wavelength using a microplate reader. At the same time, cellular morphologic changes were observed from five random fields using a phase contrast microscope (Olympus IX73; Olympus, Tokyo, Japan; magnification, x200).

Evaluation of MDA and GSH content, GPx and SOD activities. In brief, SH-SY5Y cells were treated as described above. MDA and GSH content, SOD and GSH-Px activities were detected by MDA, GSH, GSH-Px and SOD kits, respectively. 

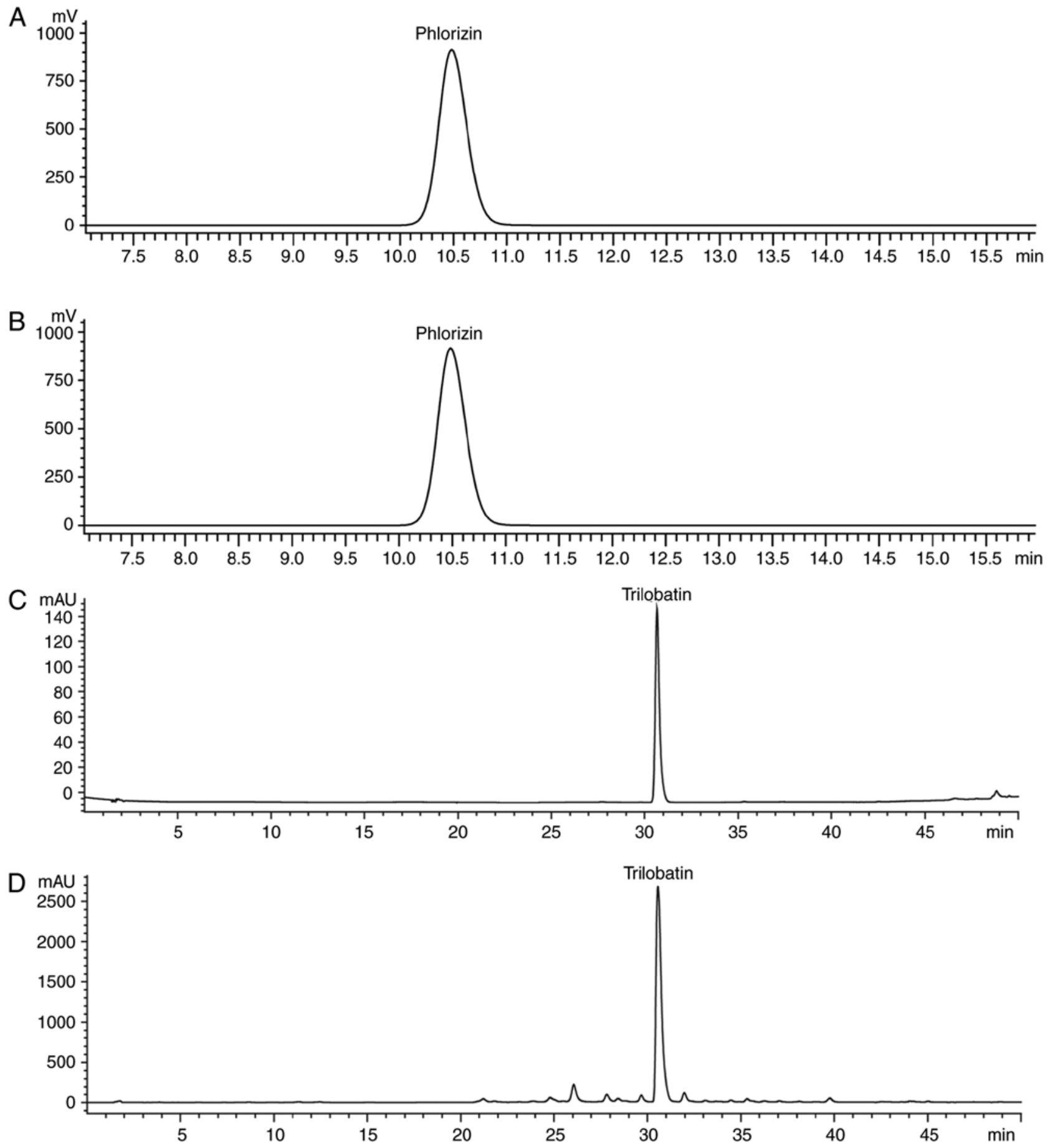

Figure 1. Representative HPLC chromatogram of phlorizin and trilobatin. (A) Phlorizin standard and (B) Phlorizin of ST. Phlorizin was detected at approximately $10.5 \mathrm{~min}$ in this system. ST also produced a phlorizin peak at this time, which corresponded to a phlorizin level of $1.19 \%$. (C) Trilobatin standard and (D) Trilobatin of ST. Trilobatin was detected at approximately $30.5 \mathrm{~min}$ in this system. ST also produced a trilobatin peak at this time, which corresponded to a trilobatin level of $18.54 \%$. ST, sweet tea.

Measurement of intracellular and mitochondrial ROS accumulation. Intracellular ROS production was measured using DCFH-DA dye. Following SH-SY5Y cells were treated for $24 \mathrm{~h}$ as described above, $20 \mu \mathrm{M}$ DCFH-DA dye treated cells for $30 \mathrm{~min}$ at $37^{\circ} \mathrm{C}$ in the dark. Subsequently, cells were washed with PBS, and intracellular ROS generation was measured using a fluorescence reader at excitation/emission wavelengths of 485/530 nm (Varioskan Flash Multimode Reader; Thermo Fisher Scientific, Inc.). Additionally, mitochondrial ROS was determined using MitoSOX Red, a fluorescent indicator for mitochondrial superoxide (18). In brief, SH-SY5Y cells were treated as described above and washed with balanced salt solution (Gibco). MitoSOX Red dye was used at a concentration of $5 \mu \mathrm{M}$ for $20 \mathrm{~min}$ in the dark at $37^{\circ} \mathrm{C}$. Subsequently, cells were washed using PBS and labeled with Mito Tracker green probe (200 nM) and stained for $20 \mathrm{~min}$. Finally, cells were washed with PBS and fluorescence was observed over the whole field of vision using fluorescence microscopy (Olympus IX73; 

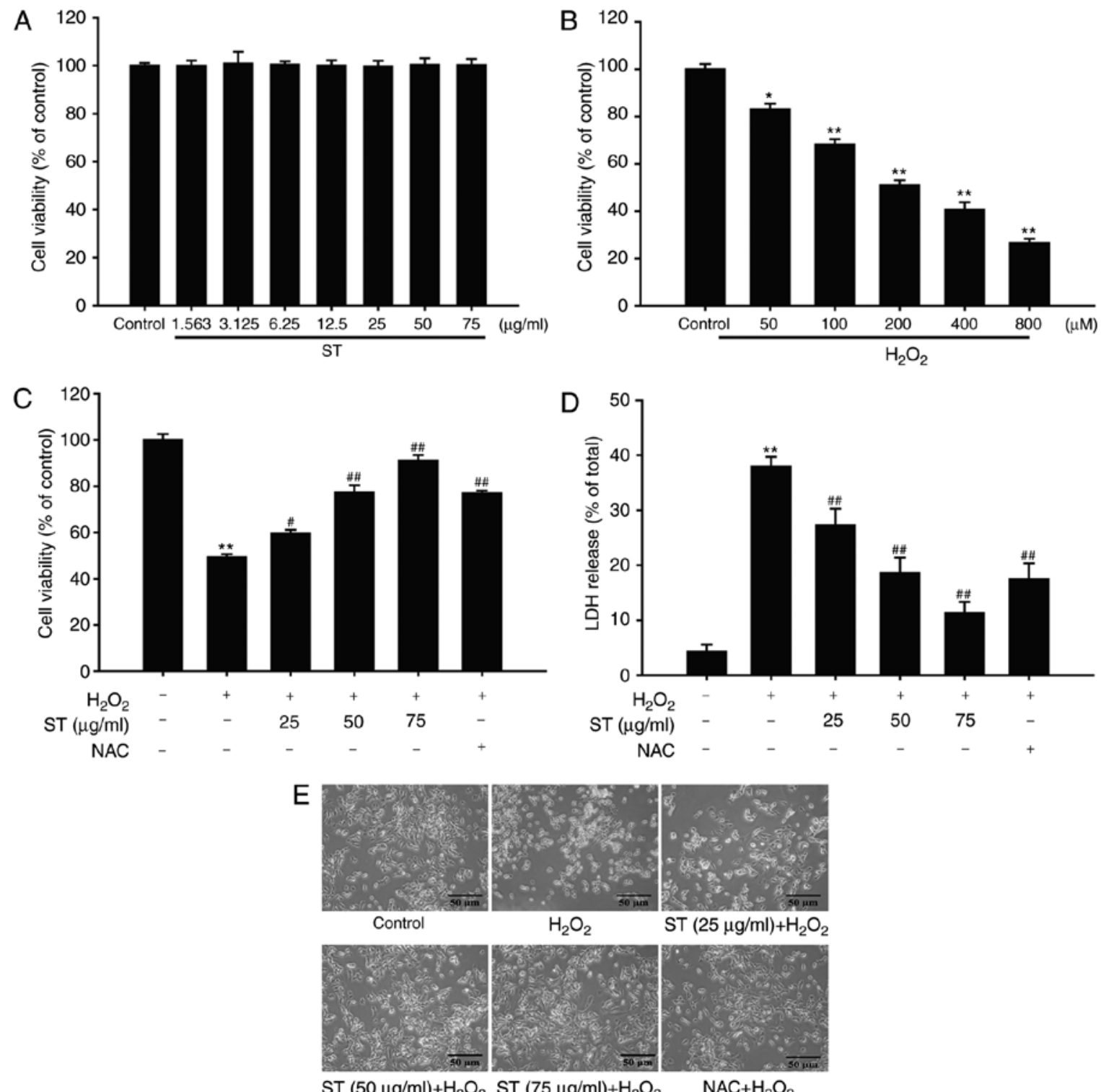

Figure 2. ST inhibits $\mathrm{H}_{2} \mathrm{O}_{2}$-induced injury in SH-SY5Y cells. (A) Cells were treated with various concentrations of ST and (B) with various concentrations

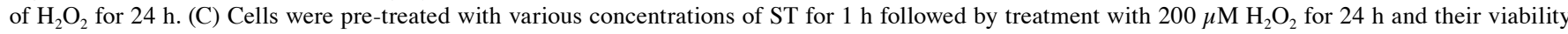
was measured by MTT. (D) LDH release from SH-SY5Y cells was determined by an LDH release assay. (E) The protective effect of $\mathrm{ST}_{\text {on }} \mathrm{H}_{2} \mathrm{O}_{2}$-induced morphological changes in SH-SY5Y cells. Data are presented as mean \pm deviation of three independent experiments. "P<0.05, * $\mathrm{P}<0.01$ vs. untreated control cells; ${ }^{\#} \mathrm{P}<0.05,{ }^{\# \#} \mathrm{P}<0.01$ vs. $\mathrm{H}_{2} \mathrm{O}_{2}$-treated cells. NAC, N-acetylcystein; ST, sweet tea.

Olympus Corporation, Tokyo, Japan; magnification, x200) with excitation/emission $(510 / 580 \mathrm{~nm})$ filters. The mean fluorescence intensity (MFI) of MitoSOX Red from five random fields was calculated using Image J 1.47i software, and the MFI was applied as an index of the levels of mitochondrial ROS.

Measurement of mitochondrial membrane potential (MMP). Measurement of MMP was detected using rhodamine 123 dye, a reliable indicator of MMP, as reported in previous studies $(19,20)$. Briefly, SH-SY5Y cells were treated as described above. Subsequently, cells were stained using rhodamine with $2 \mu \mathrm{g} / \mathrm{ml} 123$ dye for $20 \mathrm{~min}$ at $37^{\circ} \mathrm{C}$ in the dark, and cells were washed with PBS. Subsequently, fluorescence was observed over the whole field of vision using fluorescence microscopy (Olympus IX73; Olympus Corporation; magnification, x200) with excitation/emission (485/595 nm) filters.
The MFI of Rh123 from five random fields was calculated using ImageJ $1.47 \mathrm{i}$ software, and the MFI was applied as an index of the levels of MMP.

$N A D^{+} / N A D H$ measurement. The SH-SY5Y cells were treated as described above. By the end of treatment, NAD ${ }^{+} / \mathrm{NADH}$ ratio were measured using a NAD/NADH Quantification kit (cat. no. ab65348; Abcam) according to the manufacture's protocol, and absorbance was measured at $450 \mathrm{~nm}$ using the Varioskan Flash Multimode Reader.

Western blot analysis. Following pre-treatment with or without various concentrations $(25,50$ and $75 \mu \mathrm{g} / \mathrm{ml})$ of ST on $\mathrm{SH}-\mathrm{SY} 5 \mathrm{Y}$ cells for $1 \mathrm{~h}$ at $37^{\circ} \mathrm{C}$ prior to co-culture with $200 \mu \mathrm{M}$ $\mathrm{H}_{2} \mathrm{O}_{2}$. Following $24 \mathrm{~h}$ incubation, cells were lysed in the lysis buffer (cat. no. 9803; Cell Signaling Technology), and protein 

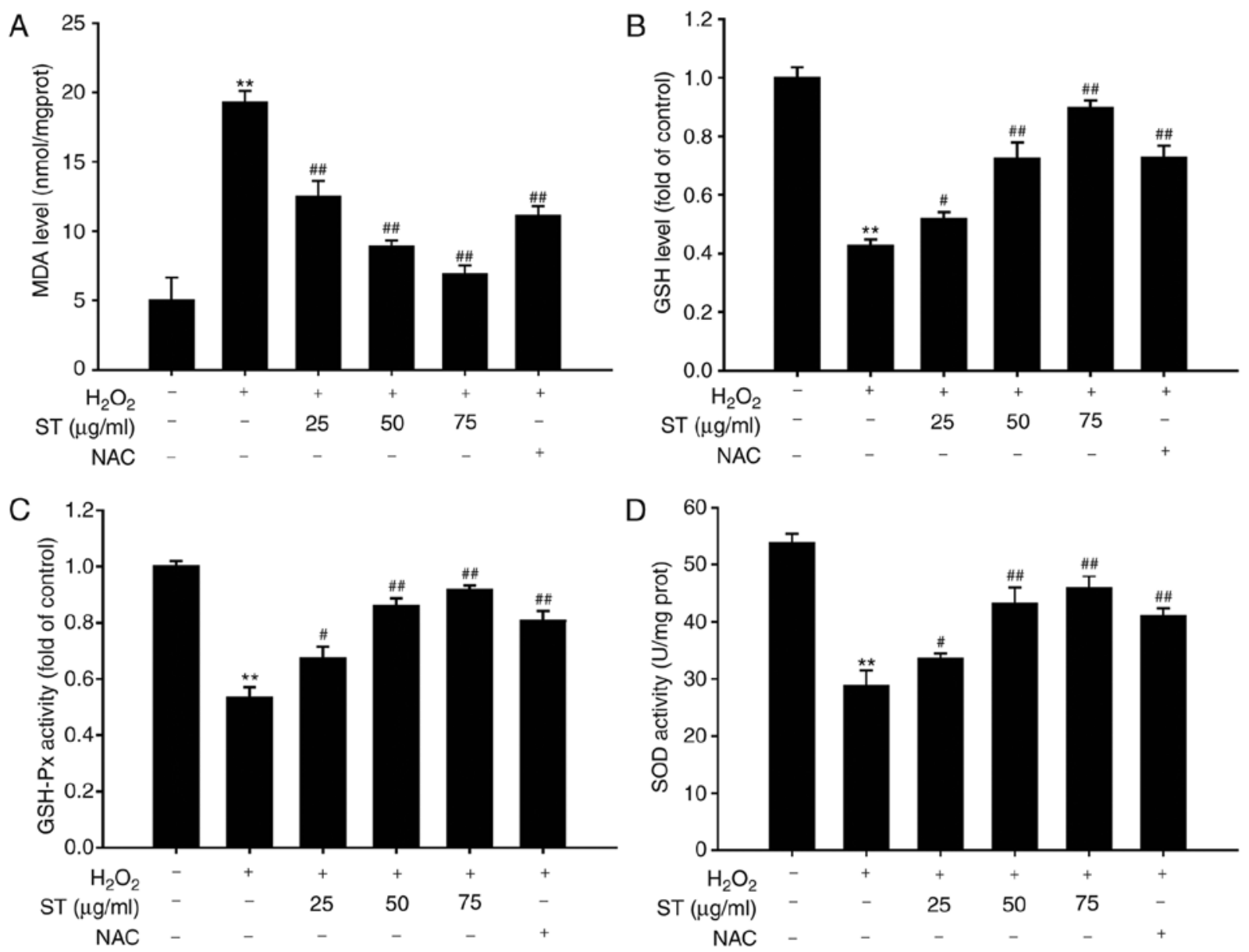

Figure 3. Effect of ST on $\mathrm{H}_{2} \mathrm{O}_{2}$-induced antioxidant enzyme activities. The cells were treated with $200 \mu \mathrm{M} \mathrm{H}_{2} \mathrm{O}_{2}$ in the presence or absence of ST for 24 h. Levels of (A) MDA; (B) GSH; (C) GSH-Px; (D) SOD activity were measured. Data are presented as the mean \pm standard error of the mean ( $\mathrm{n}=3$ ). ${ }^{*} \mathrm{P}<0.05$, ${ }^{* *} \mathrm{P}<0.01$ vs. untreated control cells; ${ }^{\#} \mathrm{P}<0.05,{ }^{\# \#} \mathrm{P}<0.01$ vs. $\mathrm{H}_{2} \mathrm{O}_{2}$-treated cells. GSH, reduced Glutathione; GSH-Px, glutathione peroxidase; MDA, malondialdehyde; NAC, N-acetylcystein; SOD, superoxide dismutase; ST, sweet tea.

concentrations were quantified using the BCA protein assay kit (cat. no. 7780; Cell Signaling Technology, Inc.). Subsequently, samples containing $20 \mu \mathrm{g}$ proteins were subjected to $10 \%$ SDS-polyacrylamide gel, and transferred to a PVDF membrane (cat. no. 1620177; Bio-Rad Laboratories). Following blocking with $5 \%$ non-fat milk for $1 \mathrm{~h}$ at room temperature, the membrane was incubated overnight at $4^{\circ} \mathrm{C}$ with the appropriate primary antibodies: anti-p-AMPK $(1: 1,000)$, anti-AMPK $(1: 1,000)$, anti-PGC-1 $\alpha$ (1:1,000), anti-Sirt3 (1:1,000), anti-IDH2 (1:1,000), anti-Foxo3a (1:1,000), anti-SOD2 (1:1,000). Subsequently, the membranes were washed with TBS with Tween-20, and incubated with appropriate HRP-conjugated goat anti-rabbit antibody (cat. no. A0208, Beyotime) and HRP-conjugated goat anti-rat antibody (cat. no. ab97057; Abcam) for $1 \mathrm{~h}$ at room temperature under shaking. The bonds of protein were developed with the ECL Western blot reagent (cat. no. P0018, Beyotime), and the blots was visualized using Davinch-Chemi ${ }^{\mathrm{TM}}$ imaging system and band optical intensity was quantified using Quantity One 1-D analysis software v4.52 (BioRad Laboratories, Inc., Hercules, CA, USA).

Statistical analysis. All data were confirmed by at least three independent experiments and were expressed as the mean \pm standard deviation. Statistical analyses were performed using SPSS 17.0 software (SPSS, Inc., Chicago, IL, USA), and individual differences were determined using one-way analysis of variance, followed by the Fisher's least significant difference. $\mathrm{P}<0.05$ was considered to indicate a statistically significant difference.

\section{Results}

ST protects $\mathrm{SH}-\mathrm{SY} 5 \mathrm{Y}$ cells against $\mathrm{H}_{2} \mathrm{O}_{2}$-induced cell injury. SH-SY5Y cells were treated with various concentrations of ST $(3.125,6.25,12.5,25,50$, and $75 \mu \mathrm{g} / \mathrm{ml})$ for $24 \mathrm{~h}$, and cell viability was subsequently detected using the MTT assay. Treatment with these concentrations of ST alone did not affect cell viability and caused no cell toxicity $[F(7,16)=0.027, P=1.000$; Fig. 2A $]$. Subsequently, the effect of $\mathrm{H}_{2} \mathrm{O}_{2}(50-800 \mu \mathrm{M})$ on SH-SY5Y cells was evaluated. The results indicated that $\mathrm{H}_{2} \mathrm{O}_{2}$ reduced cell viability in a concentration-dependent manner, and $200 \mu \mathrm{M} \mathrm{H}_{2} \mathrm{O}_{2}$ incubation with SH-SY5Y cells for $24 \mathrm{~h}$ was confirmed to be an appropriate condition to taken as an oxidative stress-induced injury in SH-SY5Y cellular model in vitro with approximately $50 \%$ cell viability inhibition rate $[F(5,12)=200.045$, $\mathrm{P}<0.001$; Fig. 2B]. To investigate the protective effect of ST against $\mathrm{H}_{2} \mathrm{O}_{2}$-induced SH-SY5Y cell injury, SH-SY5Y cells 



Figure 4. ST attenuates $\mathrm{H}_{2} \mathrm{O}_{2}$-induced mitochondrial dysfunction by inhibiting intracellular and mitochondrial ROS generation in SH-SY5Y cells. (A) The intracellular ROS level was determined with DCFH-DA dye and (B) mitochondrial ROS level was determined with MitoSOX Red dye. (C) Quantitative analysis of the MFI of MitoSOX Red in (Ba-f) using ImageJ 1.41i software. (D) Determination of MMP was carried out using rhodamine 123 dye (green). (E) Quantitative analysis of the MFI of Rh123 in (Da-f) using ImageJ 1.41i software. Data are shown as mean \pm standard error of the mean (n=3). "P<0.05, ${ }^{* *} \mathrm{P}<0.01$ vs. untreated control cells; ${ }^{\# \mathrm{P}}<0.05,{ }^{\# \#} \mathrm{P}<0.01$ vs. $\mathrm{H}_{2} \mathrm{O}_{2}$-treated cells. NAC, $\mathrm{N}$-acetylcystein; ROS, reagent active species; ST, sweet tea; MFI, mean fluorescence intensity.

were pre-treated with ST at different concentrations $(25,50$, and $75 \mu \mathrm{g} / \mathrm{ml}$ ) for $1 \mathrm{~h}$, the co-treatment with $200 \mu \mathrm{M} \mathrm{H}_{2} \mathrm{O}_{2}$ for another $24 \mathrm{~h}$. The results demonstrated that cell viability increased to $59.67,77.33,91.01$ or $76.93 \%$ when cells were pre-treated with ST $(25,50$, and $75 \mu \mathrm{g} / \mathrm{ml})$ or NAC, respectively $[\mathrm{F}(5,12)=149.1724, \mathrm{P}<0.001$; Fig. $2 \mathrm{C}]$. In parallel, $200 \mu \mathrm{M} \mathrm{H}_{2} \mathrm{O}_{2}$ significantly increased $\mathrm{LDH}$ release to $37.88 \%$. However, pre-treatment with ST or NAC, reduced the amount of LDH release to $27.23,18.55,11.29$ or $17.53 \%$ respectively $[F(5,12)=75.218, P<0.001$; Fig. $2 D]$. At the meantime, this protective effect of ST was also evidenced by morphologic observations of SH-SY5Y cells. In the case of $\mathrm{H}_{2} \mathrm{O}_{2}$-treated cells, most cells demonstrated shrinkage and floatation. However, the SH-SY5Y cells were pre-treated with ST or NAC displayed slightly morphological changes (Fig. 2E).

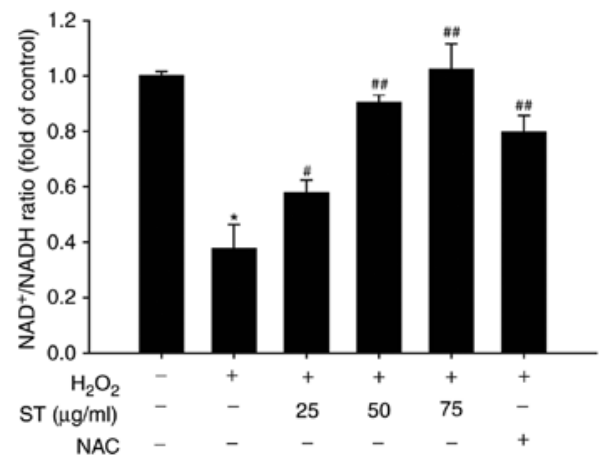

Figure 5. ST increases $\mathrm{NAD}^{+} / \mathrm{NADH}$ ratio. $\mathrm{NAD}^{+} / \mathrm{NADH}$ ratio was detected using a NADH/NAD Quantification kit. Data are shown as mean \pm standard error of the mean $(\mathrm{n}=3) .{ }^{*} \mathrm{P}<0.05,{ }^{* *} \mathrm{P}<0.01 \mathrm{vs}$. untreated control cells; ${ }^{*} \mathrm{P}<0.05$, ${ }^{\# \#} \mathrm{P}<0.01$ vs. $\mathrm{H}_{2} \mathrm{O}_{2}$-treated cells. NAC, $\mathrm{N}$-acetylcystein; ST, sweet tea. 

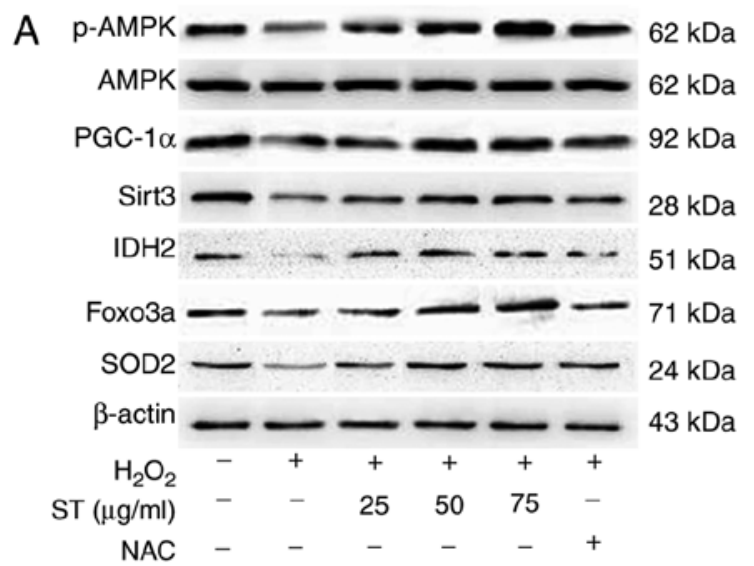

C

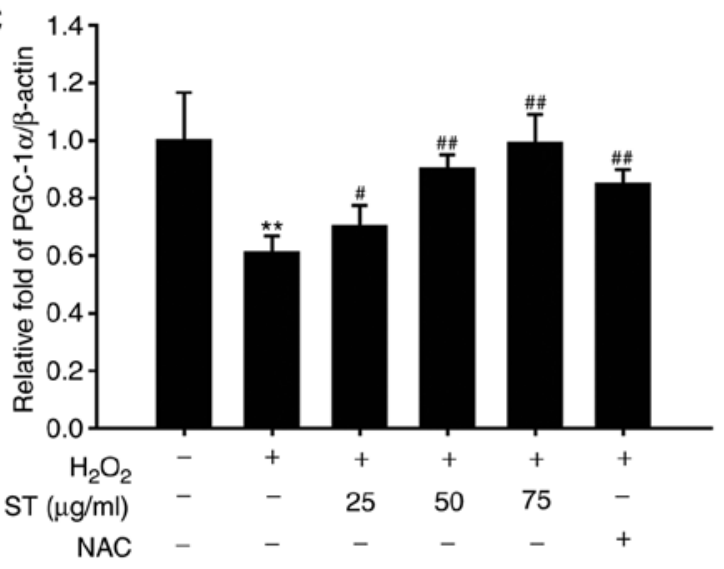

E



NAC
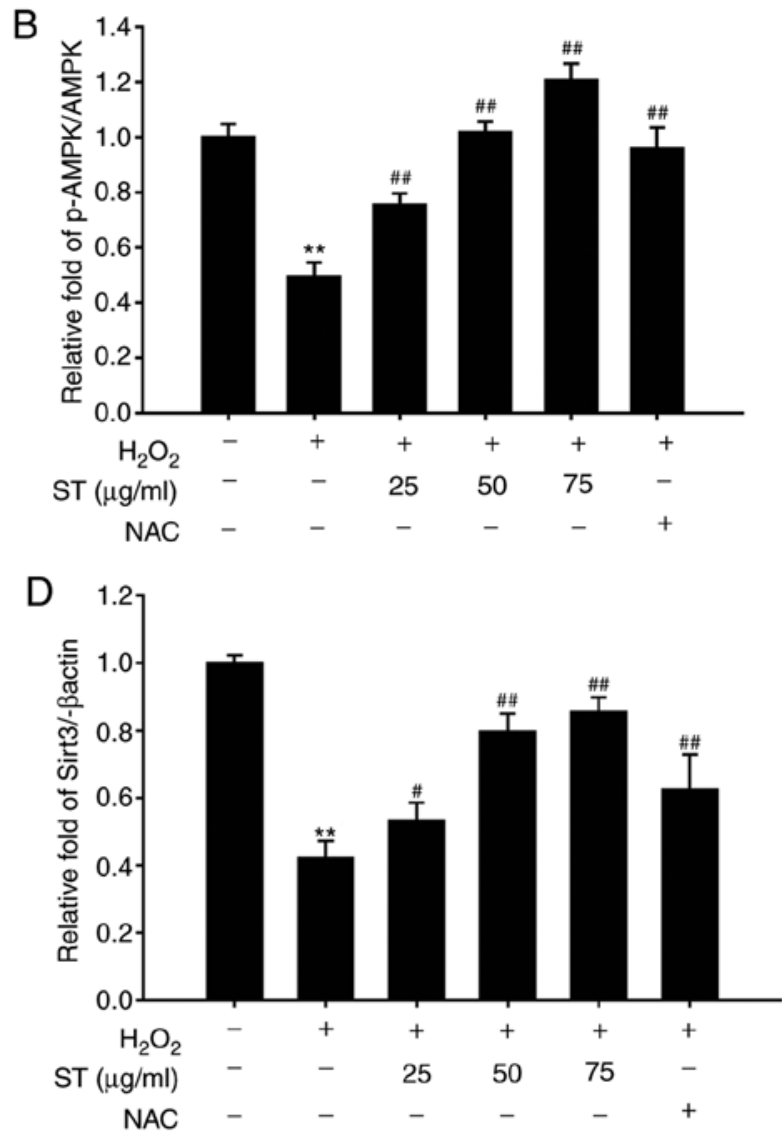

F

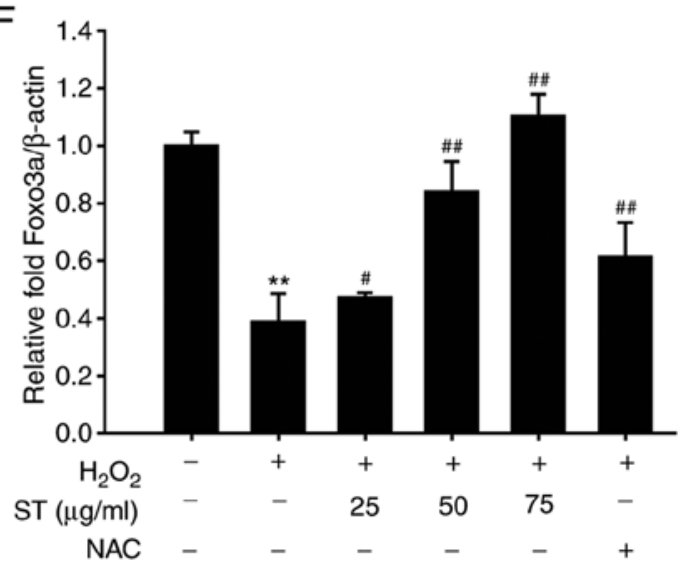

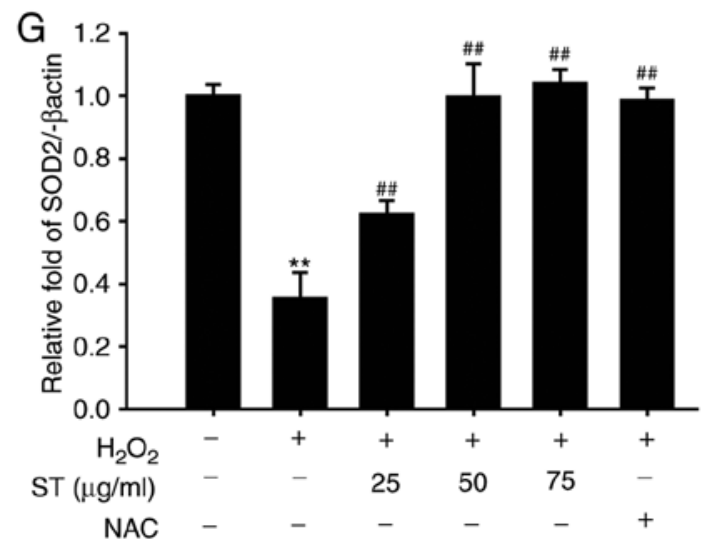

Figure 6. ST stimulates Sirt3 signaling pathway in SH-SY5Y cells. (A) Western blot analysis of AMPK, p-AMPK, PGC-1 $\alpha$, Sirt3, IDH2, Foxo3a, and SOD2 and quantification of (B) p-AMPK/AMPK ratio; (C) PGC-1 $\alpha$; (D) Sirt3; (E) IDH2; (F) Foxo3a and of (G) SOD2 protein expression levels. Data are shown as mean \pm standard error of the mean $(n=3)$. ${ }^{*} \mathrm{P}<0.05,{ }^{* *} \mathrm{P}<0.01$ vs. untreated control cells; ${ }^{\#} \mathrm{P}<0.05,{ }^{\# \#} \mathrm{P}<0.01$ vs. $\mathrm{H}_{2} \mathrm{O}_{2}$-treated cells. AMPK, adenosine monophosphate-activated protein kinase; Foxo3a, forkhead box O3a; IDH2, isocitrate dehydrogenase; p, phosho; PGC-1 $\alpha$, peroxisome proliferator-activated receptor coactivator-1 $\alpha$; ST, sweet tea. 
These findings indicated that the protective effects of ST were equal or higher than those exerted by an equivalent concentration of positive control agent.

ST attenuated $\mathrm{H}_{2} \mathrm{O}_{2}$-induced accumulation of MDA, enhanced GSH content, GSH-Px, and SOD activities. The results indicated that $\mathrm{H}_{2} \mathrm{O}_{2}$ caused a remarkable increase in MDA content and decrease in GSH content, SOD and GSH-Px activities. However, pre-treatment with ST $(25,50$, and $75 \mu \mathrm{g} / \mathrm{ml})$ significantly reduced the increase in MDA content induced by $\mathrm{H}_{2} \mathrm{O}_{2}[\mathrm{~F}(5,12)=77.225, \mathrm{P}<0.001$; Fig. $3 \mathrm{~A}]$, while it significantly reversed the decrease in GSH content, GSH-Px and SOD activities induced by $\mathrm{H}_{2} \mathrm{O}_{2}$ treatment in a concentration-dependent manner $[\mathrm{F}(5,12)=131.886, \mathrm{P}<0.001 ; \mathrm{F}(5,12)=57.409, \mathrm{P}<0.001$; $F(5,12)=97.466, P<0.001$, respectively; Fig. 3B-D].

ST attenuated $\mathrm{H}_{2} \mathrm{O}_{2}$-induced accumulation of intracellular and mitochondrial ROS production, improved MMP. Effects of ST on intracellular, mitochondrial ROS production, and MMP were evaluated. The results demonstrated that following treatment with $\mathrm{H}_{2} \mathrm{O}_{2}$, the levels of intracellular ROS increased markedly within $24 \mathrm{~h}$. Pre-treatment with ST $(25,50$, and $75 \mu \mathrm{g} / \mathrm{ml}$ ) suppressed $\mathrm{H}_{2} \mathrm{O}_{2}$-induced excessive ROS production $[F(5,12)=154.603, P<0.001$; Fig. 4A]. Furthermore, to determine the level of oxidative stress in mitochondria during $\mathrm{H}_{2} \mathrm{O}_{2}$ treatment, SH-SY5Y cells were stained with MitoSOX, a cationic probe that distributes to the mitochondrial matrix and specifically measures superoxide anion, and co-localizes with Mito Tracker Green (21). Compared with the control, $\mathrm{H}_{2} \mathrm{O}_{2}$ notably accelerated the accumulation of mitochondrial ROS which was decreased by ST pre-treatment $[\mathrm{F}(5,12)=382.644, \mathrm{P}<0.001$; Fig. 4B and C]. Furthermore, MMP indicates the function of mitochondria (22). $\mathrm{H}_{2} \mathrm{O}_{2}$ decreased rhodamine 123 fluorescence intensity, while this decrease in rhodamine 123 fluorescence was abrogated by pre-treatment with ST or NAC $[\mathrm{F}(5,12)=156.453$, $\mathrm{P}<0.001$; Fig. 4D and E]. These results demonstrated that ST effectively inhibited ROS levels and attenuated reduction in MMP induced by $\mathrm{H}_{2} \mathrm{O}_{2}$ in SH-SY5Y cells.

STincreased $N A D^{+} / N A D H$ ratio, AMPK, $P G C-1 \alpha$, Sirt3, IDH2, Foxo3a, SOD2 in SH-SY5Y cells. The results demonstrated that $\mathrm{H}_{2} \mathrm{O}_{2}$ decreased the ratio of $\mathrm{NAD}^{+} / \mathrm{NADH}$, while pre-treated with $\mathrm{ST}$ increased the ratio of NAD ${ }^{+} / \mathrm{NADH}$ in SH-SY5Y cells $[F(5,12)=91.125, P<0.001$; Fig. 5]. AMPK-PGC- $1 \alpha$ signaling serves an important role in regulating mitochondrial function and oxidative stress and has been reported to serve as the downstream target of AMPK-PGC-1 $\alpha$ signaling (23). Therefore, the effects of ST on the protein expressions of AMPK, PGC-1 $\alpha$, Sirt3, IDH2, Foxo3a, SOD2 in $\mathrm{H}_{2} \mathrm{O}_{2}$-induced SH-SY5Y cells evaluated, using western blot analysis. The results demonstrated that $200 \mu \mathrm{M} \mathrm{H}_{2} \mathrm{O}_{2}$ decreased the ratio of $\mathrm{p}$-AMPK/AMPK and the expression of PGC-1 $\alpha$ and Sirt3. However, ST pre-treatment prominently elevated the $\mathrm{p}$-AMPK/AMPK ratio and the expression of PGC- $1 \alpha$ and Sirt3 $[F(5,12)=29.286, P<0.001$; $\mathrm{F}(5,12)=9.640, \mathrm{P}<0.001 ; \mathrm{F}(5,12)=14.183$, $\mathrm{P}<0.001$; Fig. 6A-D], indicating that the AMPK-PGC-1 $\alpha$-Sirt3 axis was crucial for the protective effects of ST on regulation of mitochondrial ROS homeostasis in SH-SY5Y cells. Considering that Sirt3 is an NAD ${ }^{+}$-dependent deacetylase, and IDH2, Foxo3a and SOD2 are major substrates of Sirt3. IDH2, Foxo3a and SOD2

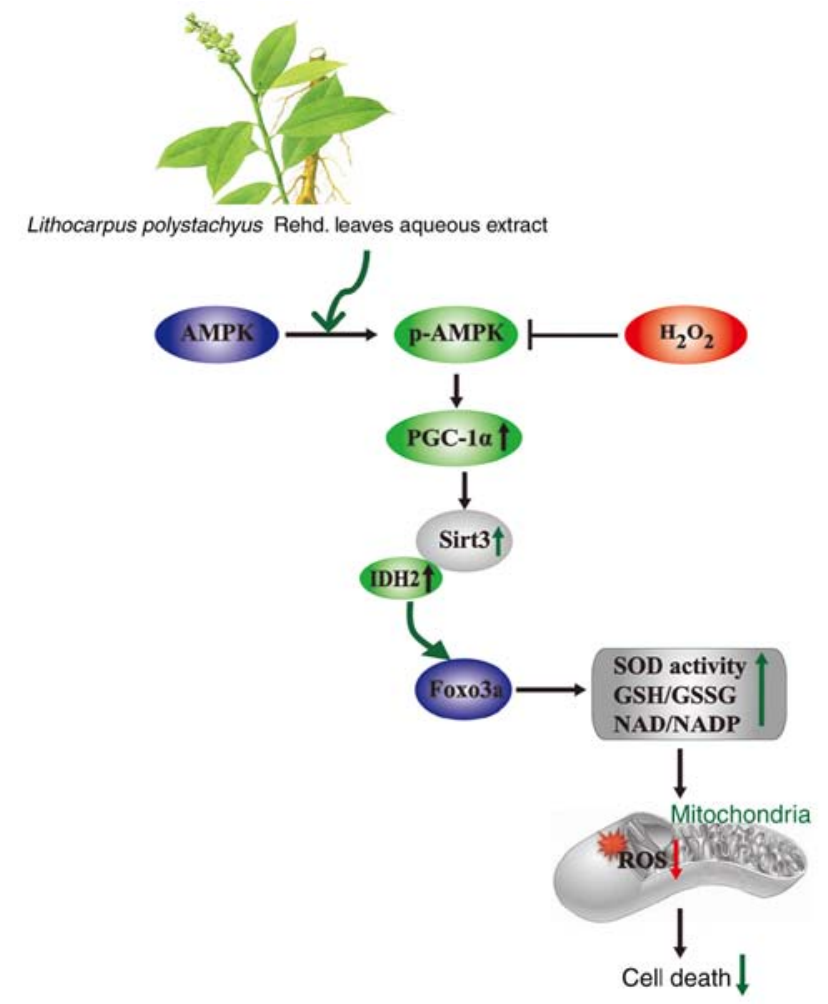

Figure 7. Model of a proposed mechanism of the protective effect of on ST SH-SY5Y cells against $\mathrm{H}_{2} \mathrm{O}_{2}$-induced SH-SY5Y cells injury through activation of AMPK-PGC-1ax $\alpha$-Sirt 3 axis. $\mathrm{H}_{2} \mathrm{O}_{2}$ is considered as the main precursor of ROS, and accumulation of intracellular ROS cause mitochondrial dysfunction, downregulation of AMPK, PGC-1 $\alpha$ and Sirt3, leading to cell death. ST reduces mitochondrial ROS production and cell death induced by $\mathrm{H}_{2} \mathrm{O}_{2}$ via mitochondrial Sirt3 signaling pathway. AMPK, adenosine monophosphate-activated protein kinase; Foxo3a, forkhead box O3a; IDH2, isocitrate dehydrogenase; $\mathrm{p}$, phosho; PGC-1 $\alpha$, peroxisome proliferator-activated receptor coactivator- $1 \alpha$; ST, sweet tea.

expressions were also investigated. The results showed that ST pre-treatment notably increased IDH2, Foxo3a and SOD2 expression compared with $\mathrm{H}_{2} \mathrm{O}_{2}$ treatment $[\mathrm{F}(5,12)=11.078$, $\mathrm{P}<0.001 ; \mathrm{F}(5,12)=20.023, \mathrm{P}<0.001 ; \mathrm{F}(5,12)=15.505, \mathrm{P}<0.001$; Fig. $6 \mathrm{~A}$ and $\mathrm{E}-\mathrm{G}]$, suggesting that $\mathrm{ST}$ ameliorated $\mathrm{H}_{2} \mathrm{O}_{2}$ induced mitochondrial dysfunction and reduced oxidative stress were mediated by the activation of AMPK-PGC- $1 \alpha$-Sirt3 signaling.

\section{Discussion}

Excessive oxidative stress has been demonstrated to serve a crucial role in neurodegenerative diseases (24). $\mathrm{H}_{2} \mathrm{O}_{2}$ not only is the most steady ROS but also serves as extracellular and intercellular messenger $(25,26)$. Therefore, in the present study, $\mathrm{H}_{2} \mathrm{O}_{2}$ was used as a toxic agent to simulate oxidative stress-induced neuronal injury in vitro, thereby leading to mitochondrial injury as described in our previous study (27). The results demonstrated that $\mathrm{H}_{2} \mathrm{O}_{2}$-induced neuronal cell injury as evidenced by decreasing cell viability and increasing cell death, combined with the repression of MMP and generation of excessive ROS in the mitochondria, which were significantly reversed by pre-treatment with ST. The present findings, for the first time to the best of our knowledge, clarified that ST exerted the protective effects on oxidative stress-induced neuronal cells injury. 
Furthermore, emerging evidence demonstrates that mitochondria are the major resource of ROS, and the mitochondrial ROS free radical scavenging systems, including SOD2 and the glutathione and thioredoxin systems, serves an key role in regulating mitochondrial ROS (28). In the present study, $\mathrm{H}_{2} \mathrm{O}_{2}$ decreased the enzymatic activities of SOD2, GSH-Px and IDH2, while these effects were reversed by pre-treatment of ST. Furthermore, MitoSOX Red, a cationic probe that distributes to the mitochondrial matrix and specifically detects superoxide, was used to determine the mitochondrial ROS as described in our previous study (27). The results suggested that ST scavenged excessive mitochondrial ROS via elevating ROS free radical scavenging systems, thereby promoted GSH levels and maintained $\mathrm{NAD}^{+} / \mathrm{NADH}$ ratio, which acts as an important role in donating electrons when NADH converts into NAD ${ }^{+}$, and eliminating excessive ROS (29). In addition, sirtuins are $\mathrm{NAD}^{+}$-dependent enzymes, which include seven homologues (Sirt1-7), and they deacetylate multiple substrates to execute numerous physiological and pathophysiological functions (30). Sirt3 is known as an important member of the sirtuin family, and it not only localizes in mitochondria but also suppresses the excessive ROS production due to deacetylating many mitochondrial proteins such as SOD2, IDH2, which improve the glutathione antioxidant defense system (31). Furthermore, the present findings indicated that $\mathrm{H}_{2} \mathrm{O}_{2}$ decreased Sirt3, Foxo3a and SOD2 expressions, which was consistent with the theory that oxidative stress downregulates Foxo3a and SOD2, which are the two main substrates of Sirt3 $(32,33)$. However, these effects were abolished by pre-treatment with ST, suggesting that ST activated Sirt3, thereby regulating Foxo3a directly and elevating SOD2 activity, which is mainly regulated by the mitochondrial sirtuin to eliminate excessive ROS (34).

Additionally, growing evidence demonstrated that Sirt3 serves as a downstream target of PGC-1 $\alpha$, which has been demonstrated as the pivotal downstream target of AMPK, thereby activating Foxo3a to reduce mitochondrial ROS production $(35,36)$. It is also postulated that decreased AMPK-PGC- $1 \alpha$ signaling pathway may lead to downregulation of Sirt3 expression (37). The results in the current study demonstrated that $\mathrm{H}_{2} \mathrm{O}_{2}$ decreased p-AMPK and PGC-1 $\alpha$ expression, which was consistent with the theory that oxidative stress inhibits AMPK, and downregulates PGC-1 $\alpha$ and Sirt3 expression. However, pre-treatment with ST reversed these effects, suggesting that ST preserved mitochondrial function and attenuated excessive ROS through activating the phosphorylation of AMPK, thereby upregulating PGC-1 $\alpha$ and Sirt3. Therefore, it can be concluded that the AMPK-PGC-1 $\alpha$-Sirt3 signaling pathway serves a crucial role in regulating mitochondrial ROS homeostasis by ST pre-treatment in oxidative stress-induced neuronal cell injury.

To conclude, the present findings indicated that ST protects against oxidative stress-induced neuronal cell injury in vitro through attenuating mitochondrial ROS, at least partly, through activating Sirt3 signaling pathway (Fig. 7). These results provide valuable insights for the role of ST, which may lead to its development as a natural antioxidant to protect neurodegenerative diseases, but this needs to be further investigated.

\section{Acknowledgements}

Not applicable.

\section{Funding}

The present study was supported by Natural Science Foundation of China (grant no. 81560666), Program for excellent young talents of Zunyi Medical Uiverstity (grant no. 15zy-002). Science and Technology Innovation Talent Team of Guizhou Province (grant no. 20154023). The 'hundred' level of high-level innovative talents in Guizhou Province (grant no. QKHRCPT 20165684), and Program for Changjiang Scholars and Innovative Research Team in University, China (grant no. IRT17R113).

\section{Availability of data and materials}

The analyzed data sets generated during the study are available from the corresponding author on reasonable request.

\section{Authors' contributions}

JG performed MTT assay, LDH leakage assay, MDA, GSH content, GPx, SOD activities analysis, ROS production, MMP analysis and was major contributor in writing the manuscript. ST aqueous extracts experiment was conducted by YX. JZ performed the western blot analysis. JS and QG designed the experimental approaches. All authors read and approved the final manuscript.

\section{Ethics approval and consent to participate}

Not applicable.

\section{Patient consent for publication}

Not applicable.

\section{Competing interests}

The authors declare they have no competing interests.

\section{References}

1. Liu L, Zhang K, Sandoval H, Yamamoto S, Jaiswal M, Sanz E, Li Z, Hui J, Graham BH Quintana A and Bellen HJ: Glial lipid droplets and ROS induced by mitochondrial defects promote neurodegeneration. Cell 160: 177-190, 2015.

2. Zhang JX, Wang R, Xi J, Shen L, Zhu AY, Qi Q, Wang QY, Zhang LJ, Wang FC, Lü HZ and Hu JG: Morroniside protects SK-N-SH human neuroblastoma cells against $\mathrm{H}_{2} \mathrm{O}_{2}$-induced damage. Int J Mol Med 39: 603-612, 2017.

3. Hanschmann EM, Godoy JR, Berndt C, Hudemann C and Lillig CH: Thioredoxins, glutaredoxins, and peroxiredoxins-molecular mechanisms and health significance: From cofactors to antioxidants to redox signaling. Antioxid Redox Signal 19: 1539-1605, 2013.

4. Tseng AH, Shieh SS and Wang DL: SIRT3 deacetylates FOXO3 to protect mitochondria against oxidative damage. Free Radic Biol Med 63: 222-234, 2013.

5. Wang Y, Wang Y, Li J, Hua L, Han B, Zhang Y, Yang X, Zeng Z, Bai $\mathrm{H}$, Yin $\mathrm{H}$ and Lou J: Effects of caffeic acid on learning deficits in a model of Alzheimer's disease. Int J Mol Med 38: 869-875, 2016

6. Wang J, Huang Y, Li K, Chen Y, Vanegas D, McLamore ES and Shen Y: Leaf extract from Lithocarpus polystachyus Rehd. promote glycogen synthesis in T2DM mice. PLoS One 11: e0166557, 2016. 
7. Hou SZ, Chen SX, Huang S, Jiang DX, Zhou CJ, Chen CQ, Liang YM and Lai XP: The hypoglycemic activity of Lithocarpus polystachyus Rehd. leaves in the experimental hyperglycemic rats. J Ethnopharmacol 138: 142-149, 2011.

8. Hou SZ, Xu SJ, Jiang DX, Chen SX, Wang LL, Huang S and Lai XP: Effect of the flavonoid fraction of Lithocarpus polystachyus Rehd. on spontaneously hypertensive and normotensive rats. J Ethnopharmacol 143: 441-447, 2012.

9. Zhou CJ, Huang S, Liu JQ, Qiu SQ, Xie FY, Song HP, Li YS, Hou SZ and Lai XP: Sweet tea leaves extract improves leptin resistance in diet-induced obese rats. J Ethnopharmacol 145: 386-392, 2013

10. Gao XY, Wang SN, Yang XH, Lan WJ, Chen ZW, Chen JK, Xie JH, Han YF, Pi RB and Yang XB: Gartanin protects neurons against glutamate-induced cell death in HT22 cells: Independence of Nrf-2 but involvement of HO-1 and AMPK. Neurochem Res 41: 2267-2277, 2016.

11. Cheng L, Li B, Chen X, Su J, Wang H, Yu S and Zheng Q: CTRP9 induces mitochondrial biogenesis and protects high glucose-induced endothelial oxidative damage via AdipoR1-SIRT1-PGC-1 $\alpha$ activation. Biochem Biophys Res Commun 477: 685-691, 2016.

12. Kincaid B and Bossy-Wetzel E: Forever young: SIRT3 a shield against mitochondrial meltdown, aging, and neurodegeneration. Front Aging Neurosci 5: 48, 2013.

13. Guan Y, Cui ZJ, Sun B, Han LP, Li CJ and Chen LM: Celastrol attenuates oxidative stress in the skeletal muscle of diabetic rats by regulating the AMPK-PGC1 $\alpha$-SIRT3 signaling pathway. Int J Mol Med 37: 1229-1238, 2016.

14. Wang Q, Li L, Li CY, Pei Z, Zhou M and Li N: SIRT3 protects cells from hypoxia via PGC- $1 \alpha$ - and MnSOD-dependent pathways. Neuroscience 286: 109-121, 2015.

15. Rangarajan P, Karthikeyan A, Lu J, Ling EA and Dheen ST: Sirtuin 3 regulates Foxo3a-mediated antioxidant pathway in microglia. Neuroscience 311: 398-414, 2015.

16. Dong H, Ning Z, Yu L, Li L, Lin L and Huang J: Preparative separation and identification of the flavonoid phlorhizin from the crude extract of Lithocarpus polystachyus Rehd. Molecules 12 : 552-562, 2007.

17. Chen TY, Chi KH, Wang JS, Chien CL and Lin WW: Reactive oxygen species are involved in FasL-induced caspase-independent cell death and inflammatory responses. Free Radic Biol Med 46: 643-655, 2009.

18. Ishii T, Takanashi Y, Sugita K, Miyazawa M, Yanagihara R, Yasuda K, Onouchi H, Kawabe N, Nakata M, Yamamoto Y, et al: Endogenous reactive oxygen species cause astrocyte defects and neuronal dysfunctions in the hippocampus: A new model for aging brain. Aging Cell 16: 39-51, 2017.

19. Gao JM, Li R, Zhang L, Jia LL, Ying XX, Dou DQ, Li JC and Li HB: Cuscuta chinensis seeds water extraction protecting murine osteoblastic MC3T3-E1 cells against tertiary butyl hydroperoxide induced injury. J Ethnopharmacol 148: 587-595, 2013.

20. Yang YY, Sun XT, Li ZX, Chen WY, Wang X, Liang ML, Shi H, Yang ZS and Zeng WT: Protective effect of angiotensin-(1-7) against hyperglycaemia-induced injury in $\mathrm{H} 9 \mathrm{c} 2$ cardiomyoblast cells via the PI3KAkt signaling pathway. Int J Mol Med 41: 1283-1292, 2018

21. Roelofs BA, Ge SX, Studlack PE and Polster BM: Low micromolar concentrations of the superoxide probe MitoSOX uncouple neural mitochondria and inhibit complex IV. Free Radic Biol Med 86: 250-258, 2015.

22. Lee Y, Heo G, Lee KM, Kim AH, Chung KW, Im E, Chung HY and Lee J: Neuroprotective effects of 2,4-dinitrophenol in an acute model of Parkinson's disease. Brain Res 1663: 184-193, 2017.
23. Hong YA, Lim JH, Kim MY, Kim Y, Park HS, Kim HW, Choi BS, Chang YS, Kim HW, Kim TY and Park CW: Extracellular superoxide dismutase attenuates renal oxidative stress through the activation of adenosine monophosphate-activated protein kinase in diabetic nephropathy. Antioxid Redox Signal 28: 1543-1561, 2018.

24. Gao C, Chang P, Yang L, Wang Y, Zhu S, Shan H, Zhang M and Tao L: Neuroprotective effects of hydrogen sulfide on sodium azide-induced oxidative stress in PC12 cells. Int J Mol Med 41: 242-250, 2018.

25. Di Domenico F, Barone E, Perluigi M and Butterfield DA: The triangle of death in Alzheimer's disease brain: The aberrant cross-talk among energy metabolism, mammalian target of rapamycin signaling, and protein homeostasis revealed by redox proteomics. Antioxid Redox Signal 26: 364-387, 2017.

26. Wang X, Dong W, Yuan B, Yang Y, Yang D, Lin X, Chen C and Zhang W: Vitamin E confers cytoprotective effects on cardiomyocytes under conditions of heat stress by increasing the expression of metallothionein. Int J Mol Med 37: 1429-1436, 2016.

27. Gao J, Deng Y, Yin C, Liu Y, Zhang W, Shi J and Gong Q: Icariside II, a novel phosphodiesterase 5 inhibitor, protects against $\mathrm{H}_{2} \mathrm{O}_{2}$-induced $\mathrm{PC} 12$ cells death by inhibiting mitochondria-mediated autophagy. J Cell Mol Med 21: 375-386, 2017.

28. Zhong J, Xu C, Gabbay-Benziv R, Lin X and Yang P: Superoxide dismutase 2 overexpression alleviates maternal diabetes-induced neural tube defects, restores mitochondrial function and suppresses cellular stress in diabetic embryopathy. Free Radic Biol Med 96: 234-244, 2016.

29. Rottenberg H and Hoek JB: The path from mitochondrial ROS to aging runs through the mitochondrial permeability transition pore. Aging Cell 16: 943-955, 2017.

30. Zhang JY, Deng YN, Zhang M, Su H and Qu QM: SIRT3 acts as a neuroprotective agent in rotenone-induced Parkinson cell model. Neurochem Res 41: 1761-1773, 2016.

31. Yin J, Han P, Tang Z, Liu Q and Shi J: Sirtuin 3 mediates neuroprotection of ketones against ischemic stroke. J Cereb Blood Flow Metab 35: 1783-1789, 2015.

32. Zhou X, Chen M, Zeng X, Yang J, Deng H, Yi L and Mi MT: Resveratrol regulates mitochondrial reactive oxygen species homeostasis through Sirt3 signaling pathway in human vascular endothelial cells. Cell Death Dis 5: e1576, 2014.

33. Guo Q, Li S, Xie Y, Zhang Q, Liu M, Xu Z, Sun H and Yang Y: The NAD ${ }^{+}$-dependent deacetylase, Bifidobacterium longum Sir2 in response to oxidative stress by deacetylating $\mathrm{SigH}$ (sigmaH) and FOXO3a in Bifidobacterium longum and HEK293T cell respectively. Free Radic Biol Med 108: 929-939, 2017.

34. Guo Y, Li Z, Shi C, Li J, Yao M and Chen X: Trichostatin A attenuates oxidative stress-mediated myocardial injury through the FoxO3a signaling pathway. Int J Mol Med 40: 999-1008, 2017.

35. Yu L, Gong B, Duan W, Fan C, Zhang J, Li Z, Xue X, Xu Y, Meng D, Li B, et al: Melatonin ameliorates myocardial ischemia/reperfusion injury in type 1 diabetic rats by preserving mitochondrial function: Role of AMPK-PGC-1 $\alpha$-SIRT3 signaling. Sci Rep 7: 41337, 2017.

36. Dai SH, Chen T, Wang YH, Zhu J, Luo P, Rao W, Yang YF, Fei $Z$ and Jiang XF: Sirt3 attenuates hydrogen peroxide-induced oxidative stress through the preservation of mitochondrial function in HT22 cells. Int J Mol Med 34: 1159-1168, 2014

37. Karnewar S, Neeli PK, Panuganti D, Kotagiri S, Mallappa S, Jain N, Jerald MK and Kotamraju S: Metformin regulates mitochondrial biogenesis and senescence through AMPK mediated H3K79 methylation: Relevance in age-associated vascular dysfunction. Biochim Biophys Acta 1864: 1115-1128, 2018. 